\title{
Capacidades estatales: un análisis para la construcción de Estado y paz en Florida y Pradera (Valle)
}

\author{
State Capacities: An Analysis for State and Peace Building in Florida \\ and Pradera (Valle) \\ Capacidades estatais: uma análise para a construção de Estado e paz \\ em Florida e Pradera (Valle)
}

\author{
SeBASTIÁN ACOSTA ZAPATA ${ }^{*}$ \\ JORGE ENRIQUE ARIAS CALDERÓN**
}

FECHA DE RECEPCIÓN: 10 DE JUNIO DE 2019. FECHA DE APROBACIÓN: 23 DE NOVIEMBRE DE 2019

Doi: https://doi.org/10.12804/revistas.urosario.edu.co/sociojuridicos/a.7988

Para citar: Acosta Zapata, S., E Arias Calderón, J. E. (2020). Capacidades estatales: un análisis para la construcción de Estado y paz en Florida y Pradera (Valle). Estudios Socio-jurídicos, 22(2), 179-208. Doi: https://doi.org/10.12804/ revistas.urosario.edu.co/sociojuridicos/a.7988

\section{RESUMEN}

Colombia, a raíz del Acuerdo de Paz firmado entre el gobierno nacional y las FARC-EP, se enfrenta a nuevos retos, como la construcción de paz y la construcción de Estado. Es, por lo tanto, que se hace necesario indagar sobre los tipos de capacidades estatales que debe desarrollar la institucionalidad para garantizar mayor eficacia y eficiencia en sus acciones, así como la posibilidad de generar confianza y credibilidad en los territorios que han soportado el conflicto armado. Este artículo tiene por objetivo principal analizar las capacidades estatales internas y externas en una perspectiva relacional en dos municipios del Valle del Cauca -Florida y Pradera- para entender las oportunidades y dificultades de la construcción de Estado, desarrollo y paz. La metodología se aborda desde un enfoque constructivista cualitativo, porque se indaga sobre las visiones, perspectivas y consideraciones que tienen los funcionarios públicos acerca de sus roles como burócratas y sus relaciones con sus territorios. También se pretende estudiar desde una postura crítica la dinámica particular que tienen los funcionarios públicos de Florida y Pradera, y considerar que la realidad es socialmente construida por los actores que se

* Universidad del Valle (Cali, Colombia). Sociólogo y politólogo con Énfasis en Relaciones Internacionales de la Universidad Icesi (Cali, Colombia); magíster en Políticas Públicas de la Universidad del Valle. Profesor e investigador del Grupo de Derecho, Estado y Sociedad (Gidse). Correo electrónico: sebastian.acosta@correounivalle.edu.co

** Universidad del Valle (Cali, Colombia). Abogado, especialista en Derecho Público de la Universidad Libre seccional Cali (Colombia) y doctor en Derecho Constitucional de la Universidad Complutense de Madrid (España). Profesor de la Universidad del Valle e investigador del Grupo Derecho, Sociedad y Estado (Gidse).Correo electrónico: jorge.arias@correounivalle.edu.co 
relacionan e interactúan en ella. Se le dio prioridad a los grupos focales como técnicas de investigación. Se concluye reflexionando acerca de las dificultades de generar habilidades para que la administración pública sea eficiente y coordinada, y se relacione de manera adecuada con los diferentes actores del territorio, e igualmente con la difícil implementación de políticas en los territorios.

Palabras clave: capacidades estatales, implementación de la paz, administración pública, gobierno local, territorio.

\section{ABSTRACT}

Colombia, following the Peace Agreement signed between the National Government and the FARC-EP, faces new challenges such as the construction of peace and the construction of the State. It is, therefore, necessary to inquire about the types of State capacities that the institutionality must develop to guarantee greater effectiveness and efficiency in its actions, as well as the possibility of generating trust and credibility in the territories that have supported the armed conflict. This article's main objective is to analyze the internal and external state capacities in a relational perspective in two municipalities of Valle del Cauca: Florida and Pradera, to understand the opportunities and difficulties of state building, development, and peace. The methodology used was a qualitative constructivist approach because it inquires about the visions, perspectives, and considerations that public officials have about their roles as bureaucrats and their relations with their territories. The article also intends to analyze, from a critical posture, the particular dynamics that Florida and Pradera's public officials have regarding that reality is socially constructed by the actors that interact among them and within it. Focus groups were prioritized as research techniques. The paper concludes reflecting on the difficulties of generating skills for the public administration to be efficient, coordinated, and adequately related to the different actors in the territory and difficulties from the implementation of policies in the territories.

Keywords: State capacities, peace implementation, public administration, local government, territory.

\section{RESUMO}

A Colômbia, devido ao Acordo de Paz assinado entre o Governo Nacional e as FARCEP, se enfrenta a novos desafios como a construção de Paz e a construção de Estado. É, portanto, que se faz necessário indagar sobre os tipos de capacidades estatais que deve desenvolver o carácter de instituição para garantir maior eficácia e eficiência em suas ações, assim como a possibilidade de gerar confiança e credibilidade nos territórios que têm sofrido o conflito armado. Este artigo tem por objetivo principal analisar as capacidades estatais internas e externas em uma perspetiva relacional em dois municípios de Valle del Cauca -Florida e Pradera- para entender as oportunidades e dificuldades da construção do Estado, desenvolvimento e paz. A metodologia se aborda desde um enfoque construtivista qualitativo porque indaga-se sobre as visões, perspetivas e considerações que têm os funcionários públicos acerca de seus roles como burocratas e suas relações com seus territórios. Também pretende-se analisar desde uma postura crítica a dinâmica particular que têm os funcionários públicos de Florida e Pradera, e considerar que a realidade é socialmente construída pelos atores que se relacionam e interatuam nela. Deu-se prioridade aos grupos focais como técnicas de pesquisa. Conclui-se refletindo acerca das dificuldades de gerar habilidades para que a administração pública seja eficiente e coordenada, e se relaciones de forma adequada com os diferentes atores do território, igualmente com a difícil implementação de políticas nos territórios.

Palavras-chave: capacidades estatais, implementação da paz, administração pública, governo local, território. 


\section{Introducción}

Este artículo surge a partir del proyecto de investigación "Gestión territorial para la implementación de una agenda de paz", 1 que se realiza dentro del marco del "Acuerdo final para la terminación del conflicto y la construcción de una paz estable y duradera" (Acuerdo Final), suscrito el 24 de agosto de 2016, ${ }^{2}$ en La Habana (Cuba), el cual desde un comienzo reconoce dos enfoques: el del gobierno nacional, que considera que las transformaciones que conlleva la implementación del Acuerdo deben contribuir a reversar los efectos del conflicto y cambiar las condiciones que han facilitado la persistencia de la violencia en el territorio; y el de las FARC-EP, quienes consideran que las transformaciones deben solucionar las causas históricas del conflicto, tales como el problema sin resolver de la propiedad privada de la tierra y su concentración, la exclusión del campesinado y el atraso de las comunidades campesinas que afecta de manera particular a las mujeres, niños y niñas (Acuerdo Final, 2016, p. 2).

Reversar los conflictos y la resolución de sus causas históricas implica necesariamente la presencia y la acción eficaz del Estado en todo el territorio nacional; es así como el Acuerdo Final constata el abandono de múltiples regiones, como también la carencia de una función pública eficaz; en consecuencia, la meta de la reconciliación nacional es la construcción de un nuevo paradigma de desarrollo y bienestar territorial (Acuerdo Final, 2016, p. 2).

Por otra parte, el enfoque territorial del Acuerdo Final supone reconocer y tener en cuenta las necesidades, características y particularidades económicas, sociales y culturales de los territorios y comunidades, los cuales juegan un papel protagónico con su participación activa; luego, la

1 Este proyecto de investigación tiene el código 8132 de la Convocatoria Interna de Investigación de la Universidad del Valle para el año 2017. El Grupo de Investigación Derecho, Sociedad y Estado de la Facultad de Ciencias de la Administración fue el que ganó la convocatoria para llevar a cabo este trabajo.

2 El 26 de septiembre de 2016 se realizó en Cartagena (Colombia) la firma definitiva del documento que fue consultado el 2 de octubre de ese mismo año mediante un plebiscito en el que el NO ganó por un estrecho margen. Luego de eso, se incorporaron algunos cambios exigidos por los opositores del acuerdo, logrando que el 24 de noviembre de 2016 se alcanzara una firma definitiva en el Teatro Colón de Bogotá (Colombia). 
implementación se plantea desde las regiones y territorios, y con la participación de las autoridades territoriales y los diferentes sectores sociales.

Conforme a lo anterior, el proyecto de investigación sobre el cual se realiza este artículo pretende entre uno de sus objetivos: analizar la capacidad estatal de los municipios de Pradera y Florida para una agenda de desarrollo local en el marco del Acuerdo Final para la Paz, sin embargo, este no es el objetivo general del artículo. La reflexión teórica acerca de las capacidades estatales constituye la comprensión del concepto y la necesidad de su delimitación para abordar empíricamente el análisis de las capacidades de los dos municipios antes mencionados.

En los programas de desarrollo con enfoque territorial (PDET), se definieron varias áreas de injerencia, una de las cuales es denominada "Norte del Cauca-Alto Patía", compuesta por Florida y Pradera en el Valle del Cauca, siendo estos municipios de sexta categoría, según el Departamento Nacional de Planeación,, junto con el municipio de Buenaventura; estas tres localidades en el departamento del Valle del Cauca podrán disponer de más recursos para efectuar un plan de desarrollo propio y garantizado por el Acuerdo Final.

Considerando el avance conceptual y la propuesta de la organización Dejusticia, en su publicación "Los territorios de la paz. La construcción del Estado local en Colombia" (2016), se toma en consideración la idea de que tanto Estado como sociedad civil deben participar y comprometerse con la construcción de un Estado capaz y eficiente, y con una paz estable y duradera.

Un proyecto de integración nacional y de construcción de instituciones locales necesita de un delicado balance entre, por un lado, la participación ciudadana, el compromiso comunitario y el involucramiento de los movimientos sociales y, por el otro, el acompañamiento legal y democrático (con jueces más que con Ejército) del Estado central. Por eso es necesario empezar a pensar, desde ya, en diseños institucionales adecuados que hagan frente a las complejas realidades que condicionan el éxito de este gran proyecto de paz y desarrollo. 
Las reformas que se aprueben deberán conciliar ambas miradas: las necesidades de seguridad y las necesidades de participación. Dicho de otro modo, la reforma debe lograr el mayor grado de participación posible, compatible con el mayor grado de orden posible (García, et al., 2016).

El desarrollo metodológico de este trabajo se orienta desde una perspectiva constructivista con un enfoque cualitativo, porque se indaga sobre las visiones, perspectivas y consideraciones que tienen los funcionarios públicos acerca de sus roles como burócratas y sus relaciones con sus territorios. También se pretende analizar desde una postura crítica la dinámica particular que tienen los funcionarios públicos de Florida y Pradera, y considerar que la realidad es socialmente construida por los actores que se relacionan e interactúan en ella. Acorde con las características conceptuales de las capacidades estatales, se establecen dos dimensiones o categorías de análisis: las capacidades internas y las capacidades externas. Igualmente, se abordan elementos que procuran profundizar la comprensión del concepto estudiado conforme con cada dimensión, siendo estas la burocracia, el mérito, la profesionalización y la coordinación, en cuanto a las capacidades internas. Y las relaciones con los diferentes habitantes del territorio, la confianza, la construcción de acciones participativas conjuntas, en cuanto a las capacidades externas.

Se realizó un grupo focal y entrevistas grupales a varios funcionarios y contratistas de las administraciones públicas de Florida y Pradera; en estas actividades se abordó el tema de las capacidades estatales desde su óptica y cotidianidad. El instrumento construido fue semiestructurado con rotuladores como 'relaciones', 'dificultades', 'experiencias', 'logros', 'problemas', 'confianza', 'gestión', 'desarrollo', 'paz', desarrollando ejercicios más próximos a la realidad de los participantes para no encasillar y limitar el abordaje efectuado. ${ }^{4}$

Teniendo en cuenta que el objetivo principal del presente artículo es analizar las capacidades estatales internas y externas en una perspectiva relacional en dos municipios del Valle del Cauca -Florida y Pradera- para

4 El tiempo de duración de cada entrevista grupal osciló entre 1 hora y 30 minutos y 2 horas, con un promedio de participantes de cinco personas, representantes de las diferentes secretarías y dependencias de los gobiernos municipales. 
entender las oportunidades y dificultades de la construcción de Estado, desarrollo y paz, las técnicas de investigación anteriormente mencionadas se ajustan a este en la medida que permite concebir, desde los funcionarios públicos, las dinámicas cotidianas que pueden representar las capacidades estatales en las dos dimensiones: internas y externas.

Para adelantar este estudio, en primer lugar, se aborda una reflexión conceptual general acerca de las capacidades estatales, a partir de una serie de preguntas orientadoras que guían el fenómeno de las capacidades estatales. En segundo lugar, se delimita conceptualmente la noción de capacidades estatales, junto con sus límites, proponiéndose la comprensión más detallada de estas nociones. En tercer lugar, se entra a hacer el análisis empírico establecido. Y, por último, se concluye con los aspectos más relevantes.

\section{Una reflexión conceptual general sobre las capacidades estatales}

¿Qué se entiende concretamente por capacidad estatal o institucional? ¿Cuáles son sus desarrollos conceptuales? ¿Cómo entender las capacidades estatales en sus diferentes dimensiones? ¿Cómo se operativizan los conceptos de capacidades estatales?

Una primera aproximación al tema de las capacidades estatales o institucionales se relaciona con la "capacidad como la habilidad para desempeñar tareas de manera efectiva, eficiente y sostenible" (Hilderbrand \& Grindle, 1997); esta definición aparentemente sencilla encierra una gran dificultad al momento de usarla para hacer observaciones empíricas.

A efectos de ir delimitando el concepto de capacidad institucional, Grindle (1997) sugiere que el enfoque de buen gobierno se relaciona con la capacidad del gobierno en tres dimensiones: el desarrollo del recurso humano (sistemas y procesos de personal), el fortalecimiento organizacional (sistemas de gestión) y la reforma institucional (instituciones y sistemas a nivel macro). Este enfoque que ofrece esta autora representa un punto intermedio entre una visión demasiado amplia, que equipara 'capacidad' con 'desarrollo', y una visión demasiado reducida, que la equipara con el simple desarrollo de habilidades y competencias 
en el recurso humano. El análisis de las capacidades estatales se entiende como relación entre el sector público en el interior, y de este con comunidades y territorios, es decir, con el exterior (Completa, 2016, 2017; Fernández \& Vigil, 2012; Bertranou, 2013; Rey, 2014; Oszlak \& Orellana, 1991).

También se hace necesario abordar lo que Uribe López (2018) concibe como la construcción de Estado y la construcción de paz. Así, el autor, siguiendo a Lederach (2010), plantea que la construcción de paz es un concepto comprehensivo que engloba, produce y soporta el total de procesos, líneas, enfoques y fases importantes para transformar los conflictos hacia relaciones más sostenibles, pacíficas, seguras e, incluso, institucionalizadas.

Uribe López (2018) sostiene que la articulación entre las infraestructuras de paz y gobernanza territorial dan una oportunidad de potenciar enclaves colaborativos para construir paz e institucionalidad en Colombia.

El reconocimiento de la construcción estatal y de la construcción institucional, como dimensiones fundamentales y complementarias de la construcción de paz, ha hecho del "diseño de las instituciones correctas" una suerte de mantra contemporáneo de los constructores de paz. Hay en la actualidad un acuerdo bastante amplio sobre la necesidad de contar con Estados que funcionen gracias a las instituciones apropiadas para prevenir y arbitrar, pacíficamente, los conflictos entre diferentes grupos sociales y sus expresiones políticas (Uribe López, 2018, p. 179).

Por lo tanto, es fundamental elaborar infraestructuras para la paz en sintonía con estrategias de acción colectiva, generadas desde las regiones y los territorios en una perspectiva totalmente local, en la que se avance en la construcción de relaciones tanto formales como informales con organizaciones de base, actores regionales y actores institucionales en todos los niveles, incluyendo el nacional e internacional. Toda vez que esto permite coordinar decisiones, recursos y procesos para la construcción de paz, y de Estado, que no son lo mismo pero sí tienen que ser complementarias (Uribe López, 2018).

Diversos autores, como Completa (2016, 2017), Fernández y Vigil (2012), Bertranou (2013) y Rey (2014), establecen la necesidad de 
estudiar las capacidades estatales primordiales para llevar a cabo procesos de diseño e implementación de política públicas, así como mejorar la eficiencia del Estado en la interlocución con las sociedades con las que se relaciona. El interés aumenta en la medida que el Estado latinoamericano ha sido tradicionalmente grande pero débil (Isuani, 2012), por ello requiere de estrategias de modernización como la gobernanza (Aguilar, 2010). Aguilar (2010) considera que la legitimidad del gobierno - sea nacional, regional o local-, y a su vez del Estado, radica en la eficiencia, pues el sustento, la confianza y el respaldo a la institucionalidad no depende de la forma en que fue elegido, sino de los resultados que demuestre y las capacidades que tenga para resolver los problemas sociales que se presenten.

La discusión gira sobre lo que el Estado y la institucionalidad pueden hacer. En franca tensión con las políticas de ajuste neoliberales que procuraron una reducción del Estado en la primera parte de la década de los noventa, el giro en 2000 buscó envestir al Estado de más fuerza, sin retroceder en el adelgazamiento del aparato estatal, lo que ocasionó el interés del gobierno por hacer más pertinente el servicio y la función pública: eficiencia, efectividad y capacidad de respuesta (Completa, 2016). Como complemento, Alonso (2007) plantea que el interés de los Estados en la profesionalización de la burocracia hizo palpable la brecha de capacidades estatales en lo concerniente a la carrera de la función pública.

Países como Brasil, México, Argentina y Colombia procuraron modernizar los procesos burocráticos y profesionalizar el servicio civil (Ospina, 2002), no obstante, los resultados no han sido muy satisfactorios, ya que la administración pública y las funciones del Estado en cuanto a gestión no han sido separadas plenamente de las disputas políticas y los avatares electorales. Este panorama riñe con la visión más tecnócrata y funcionalista que plantea Weber en el uso y construcción de la burocracia. Por lo tanto, no hay una división entre la administración de los asuntos públicos y la política electoral.

Si bien Colombia no dio un giro ideológico a la izquierda como lo hizo Venezuela con Hugo Chávez, Ecuador con Rafael Correa, Brasil con Luiz Ignacio Lula da Silva, Argentina con Néstor Kirchner, Chile con Michelle Bachelet, Uruguay con Tabaré Vázquez, entre otros, se 
preocupó por modernizar el Estado, la función pública y la estructura estatal. Tanto los presidentes Uribe en 2003 como Santos en 2011 impulsaron reformas político-administrativas para enfrentar los retos de la administración. No obstante, el argumento clave en la reforma de 2011 era preparar al país para el futuro, es decir, agilizar los procesos, especializar el servicio público y adecuar institucionalmente al Estado para un 'eventual posconflicto', por lo cual surgen entonces entidades como las agencias, de estructura liviana, que acompañan a los ministerios, están adscritas a la Presidencia y cuya labor más general es de orientación de las políticas públicas (Sarria, 2015).

El reto para Colombia es que su Estado no se vea extraño, ajeno, intruso ante su sociedad. El Estado busca construir lazos sociales y ganarse la confianza de las comunidades siempre olvidadas, en las que los poderes ilegales configuraron una suerte de Estado paralelo en algunos casos, o se apropiaron de funciones del Estado en las regiones más azotadas por el conflicto armado interno. ${ }^{5}$

Así, las capacidades estatales en el escenario de un 'posacuerdo' concentran la habilidad de la institucionalidad para establecer un diálogo entre ella y las comunidades en las que pretende impactar. Por lo tanto, en el marco de la implementación del "Acuerdo final para la terminación del conflicto y la construcción de una paz estable y duradera", se crearon estrategias como el diseño de los planes de desarrollo con enfoque territorial (PDET) ${ }^{6}$ a cargo de la Agencia de Renovación del Territorio (ART), que pretendía establecer un diálogo directo entre el gobierno nacional y las zonas más afectadas del conflicto armado.

5 Esta idea es especialmente poderosa en el libro La captura y reconfiguración cooptada del Estado en Colombia, cuyos autores son Luis Jorge Garay Salamanca (Dirección Académica), Eduardo Salcedo-Albarán, Isaac de León-Beltrán y Bernardo Guerrero (2008); y en la obra $Y$ refundaron la patria... de cómo mafiosos y políticos reconfiguraron el Estado colombiano, de Claudia López (2010).

6 "El PDET fue creado por el Decreto 893 de 2017. Es un programa subregional de transformación integral del ámbito rural a 10 años a través del cual se ponen en marcha con mayor celeridad los instrumentos de la RRI en los territorios más afectados por el conflicto armado, la pobreza, las economías ilícitas y la debilidad institucional. Es un instrumento de planificación y gestión para implementar de manera prioritaria los planes sectoriales y programas en el marco de la reforma rural integral y las medidas pertinentes que establece el Acuerdo Final, en los municipios priorizados" (Presidencia de Colombia, 2017). 


\section{Capacidades estatales como concepto}

\section{Estado del arte de las capacidades estatales}

En una concepción amplia, Evans (1996) teoriza que la profundidad de capacidades estatales depende, en primer término, de la medida en que el Estado logra un aislamiento burocrático en el sentido manifestado por Weber, es decir, que, mientras el funcionamiento administrativo del Estado esté más reglado, normado, protegido, cercado y aislado de las dinámicas electorales y políticas, más eficaz y eficiente es; en segundo término, es imperante un proceso en que el Estado realice una penetración en la estructura social circundante, que se muestre notorio, relevante, importante para la definición y solución de problemáticas sociales. En consecuencia, la 'autonomía enraizada' referencia una burocracia que crea y desarrolla lazos sociales concretos y reales con las comunidades, y se muestra como un canal relevante para el diálogo, la negociación, renegociación y toma de decisiones en cuanto a las formas de proceder del Estado en lo relacionado con las políticas públicas y los objetivos estratégicos trazados.

Evans (1996) señala que el Estado precisa de una serie de dinámicas tanto internas como externas que impactan sobre la estructura estatal y el desempeño de la burocracia, que resultan útiles para la consecución de metas propuestas por la institucionalidad. El Estado requiere una mezcla de coherencia interna y de interacciones externas que permitan consolidar una capacidad transformadora. Evans enfoca su perspectiva en la estructura y el desempeño mismo de la burocracia como aquel dispositivo de penetración y logros de los objetivos del poder público.

Es clave señalar la conceptualización que Weber (Gilio, 2016) hace de la burocracia al ser ese sistema de méritos en el que se combina la formación académica, la experiencia, el acceso calificado a la administración pública y la posibilidad de desarrollar una carrera profesional dentro del Estado, en el que el desempeño sea un factor crucial para los ascensos y sea un incentivo positivo que motive a los empleados públicos.

Siguiendo con la argumentación planteada por Evans y Weber, se precisa de un aparato administrativo incentivado y capacitado para lograr una gestión competente, generar coherencia entre los diferentes niveles 
del Estado y una adecuada representación de la estatalidad. Todo esto es necesario para mejorar las capacidades estatales y la inserción en la sociedad (Evans, 1996).

Por otra parte, Completa (2016) trae a colación el trabajo de Grindle (1996), quien plantea que, para que los Estados y sus agencias o administraciones tengan un buen desempeño, deben tener cuatro tipos de capacidades: 1) la capacidad institucional, como la habilidad para establecer y hacer valer las reglas que imperan sobre las interacciones políticas y económicas; 2 ) la capacidad técnica para administrar las políticas públicas; 3 ) la capacidad administrativa de los Estados y administraciones públicas para proveer de bienes y servicios a las comunidades; 4) la capacidad política entendida de los Estados para tomar en cuenta las demandas de la sociedad y responder frente a ellas.

Las capacidades estatales, concebidas como lo expresa Completa (2016), se definen como la habilidad real y potencial que los Estados, sus agencias, oficinas, tanto de alcance nacional como regional y local, deben poseer para canalizar las demandas de los diferentes actores involucrados en el escenario social, político y económico del territorio, y poder dar respuesta mediante la toma de decisiones de manera oportuna, eficaz, eficiente y autónoma, no limitándose exclusivamente por el entorno institucional, con el fin de alcanzar las metas propuestas, tanto para la organización estatal como para las comunidades, y lograr consolidar un bienestar general.

Fortaleciendo las líneas argumentativas previas, Bertranou (2013) concibe las capacidades estatales desde dos dimensiones: la espacioterritorial y la relacional. La primera es la capacidad del Estado para hacer presencia en todo el territorio que comprende, es decir, manifestarse efectivamente en todas las regiones. La segunda se decanta de los tipos de relaciones que el Estado sostiene con actores no estatales (sociedad civil, empresarios, gremios, grupos ilegales) y el grado de control que tiene sobre estos.

Continuando con Bertranou (2013), las capacidades estatales solo pueden ser analizadas y apreciadas a partir de la especificación de las funciones que un organismo debe cumplir o los objetivos que este debe alcanzar. Las capacidades estatales deben analizarse en concreto, en relación con unos fines establecidos y con metas trazadas que la 
entidad debe cumplir y lograr. El término capacidad se presenta como un concepto de carácter instrumental, como un atributo que se resignifica de acuerdo con el contexto histórico-temporal o con el paradigma en el que se encuentre inserto, factores que predeterminan los fines y metas que debe perseguir el Estado y sus agencias específicas.

Alonso (2007), profundizando la secuencia argumental expuesta, considera que las dos dimensiones que se presentan en el análisis de las capacidades estatales son la técnico-administrativa y la relacional. La primera se centra en priorizar una mirada desde adentro del Estado en todos sus niveles, o sea que el aparato estatal se integre, se comunique, se relacione entre sí, generando una identidad de cuerpo. La segunda dimensión pone el foco en la interrelación entre el Estado y la sociedad. En suma, para Alonso, las capacidades estatales se dan en dos vías, hacia adentro del Estado y hacia afuera.

La construcción de capacidades por parte del Estado se configura como recursos con habilidades extractivas, intervencionistas, reguladoras y mediadoras, son en tanto un proceso de institucionalización en el que se desarrollan y consolidan las habilidades y prácticas cotidianas por parte del Estado en general, y de la agencia pública específica en particular. Se busca la habilidad de proveer una estructuración estable y predecible para la interacción estratégica de actores (Alonso, 2007).

Tomando en cuenta la perspectiva de Mann (1984) en "El poder autónomo del Estado", se distinguen dos tipos de poderes: el despótico y el infraestructural. El primero es el que se ejerce en la lucha por el poder entre las élites y grupos de interés de otros lugares; y el segundo es el que se ejerce gracias a la provisión de servicios públicos. Estos dos tipos de poder estatal proceden de la capacidad del Estado para proporcionar una forma de organización territorialmente organizada.

Siguiendo con Mann (1984), se distingue que el Estado provee a las sociedades de reglas de convivencia y de juego relevantes para la protección de la vida y la propiedad, y el poder autónomo del Estado se deriva de la necesidad que tienen las sociedades de estas garantías de protección. Así mismo, las actividades del personal estatal, es decir, de los burócratas, son necesarias para la sociedad en su conjunto, que se beneficia de la estructura de reglas existentes. Para fines de este artículo, se toma la noción del poder infraestructural de Mann (1984), pues se 
considera que la provisión de bienes públicos, de servicios burocráticos y de construcción de paz son ejercicio del poder del Estado en cuanto a su responsabilidad de proveer servicios públicos a su sociedad.

Fernández y Vigil (2012), citando a Michael Mann (1984), hacen alusión al desarrollo de las capacidades infraestructurales del Estado para desarrollar la aptitud de penetrar en la sociedad y llevar a cabo un alto poder operativo al fortalecerse de los conocimientos, recursos, habilidades y relaciones con las poblaciones. Se concibe como un proceso de 'enraizamiento' de las agencias, entidades y administraciones públicas locales y regionales en el espacio donde interactúan los diferentes actores, sean sociales, políticos o económicos. Estas llamadas capacidades infraestructurales se complementan con las capacidades internas, es decir, con las habilidades de comunicación y de articulación entre las diferentes entidades del Estado, impidiendo que se cerquen las burocracias y los tomadores de decisiones que trabajan, se relacionan e interactúan entre ellas, y potencializando los recursos, las herramientas, los conocimientos, las aptitudes y la maniobrabilidad que pueda llegar a construir el Estado frente a un complejo escenario de diversas clases de actores de la sociedad.

Para Completa (2016), Bertranou (2013) y Rodríguez (2004), las capacidades estatales se ubican en dos tipos o dimensiones: 1) las internas, en las que se fortalece el aparato estatal hacia adentro, que busca fortificar sus relaciones intraestatales; mejorar y capacitar la burocracia, el servicio civil; generar incentivos positivos para la carrera administrativa; establecer un diálogo fluido en todos los niveles de la administración pública desde el nacional hasta el regional y el local. Estas habilidades son comúnmente descritas en la literatura como administrativas y/o institucionales. 2) Las externas, en las que se busca establecer puentes y acercamientos a los distintos actores de la sociedad; llegar a construir redes de confianza con las comunidades y con los diferentes grupos que hacen una presencia fuerte en el territorio; enfocarse en la habilidad de responder a las demandas sociales mediante acciones concretas, como planes, programas, proyectos y políticas públicas, que permitan representar al Estado como un ente político-administrativo coherente, incluso monolítico. Estas habilidades son descritas en la literatura como políticas o infraestructurales, y buscan penetrar la sociedad, ganarse su confianza y enraizarse con procesos de mejora del bienestar común. 


\section{Propuesta de la noción: capacidades estatales}

La investigación ha procurado desarrollar un concepto, no nuevo, pero sí útil, para entender el fenómeno de las capacidades estatales y poder operacionalizar los resultados obtenidos en campo. Teniendo en cuenta el numeral anterior, se concibe que las capacidades estatales se centran en las habilidades relacionales en el marco de un proceso establecido y situado como la construcción de Estado y de paz en un escenario de posacuerdo (Uribe López, 2018; Restrepo, 2018) en dos municipios que han tenido presencia diferenciada del Estado en momentos de conflicto armado, y donde han ejercido algún tipo de influencia las Fuerzas Armadas Revolucionarias de Colombia (Blanco \& Arrieta, 2019). Producto del Acuerdo Final, el gobierno nacional creó una serie de dependencias adscritas directamente a la Presidencia, las cuales tenía por finalidad agilizar la implementación de los compromisos adquiridos. Es por ello que las funciones de construcción del Estado y de paz - realizadas en formas paralelas- pertenecen a una agencia, oficina o entidad administrativa particular, y buscan cumplir una serie de objetivos de la manera más eficiente, eficaz, y con la mayor capacidad de respuesta sostenible posible. Se considera que las capacidades estatales son procesos en continua construcción y de largo aliento que solo tendrán resultados en el plazo extendido.

Considerando los avances conceptuales de Completa (2016), Bertranou (2013) y Rodríguez (2004), Fernández y Vigil (2012), Michael Mann (1984), Alonso (2007) y Evans (1996), entre otros, se entiende por capacidades estatales las habilidades relacionales que tienen las diferentes entidades públicas como representantes no monolíticos del Estado para penetrar la sociedad en la que hacen presencia, enraizar las burocracias a la confianza social y generar vínculos cotidianos en la dinámica constante de consolidar, desde un enfoque de poder infraestructural, provisión de bienes y servicios públicos. En este marco se conciben dos tipos de capacidades: las internas y las externas. Las primeras tienen que ver con las relaciones entre los diferentes actores y entidades públicas entre sí; las segundas, con las relaciones de los distintos actores y entidades públicas con los territorios, comunidades y organizaciones sociales en los cuales pretenden influir, intervenir y actuar. 
Es por ello que, para la presentación y discusión de los resultados obtenidos en campo (el subtítulo siguiente), este artículo ha buscado operacionalizar las capacidades estatales desde una doble óptica: las internas y las externas, como anteriormente se describió.

\section{Resultados: trabajo de campo, reflexión, discusión y análisis}

Este apartado cuenta con dos partes: la primera se centra en las capacidades internas de las administraciones de Florida y Pradera, y procura relatar, describir y analizar la forma en la que se establecen las relaciones dentro de estos gobiernos locales; igualmente, la articulación y coordinación entre las distintas dependencias que las conforman y la interrelación con el gobierno nacional. La segunda se concentra en las capacidades externas de los municipios aquí analizados, construyendo vínculos de confianza a fin de reducir la conflictividad y reticencia de los territorios frente a su accionar. Es transversal el análisis del aspecto relacional de los dos tipos de capacidades.

\section{Capacidades internas: entre la burocracia y las dinámicas electorales}

Un primer acercamiento a los municipios objeto de análisis tiene relación con el cumplimiento de metas propuestas en sus planes de desarrollo y en la ejecución de su presupuesto; es así como en el caso de Florida formalmente se ha logrado ejecutar el $83 \%$ de su Plan de Desarrollo Municipal 2016-2019 a febrero de 2019, teniendo en cuenta que la presente administración finaliza en diciembre de 2019, esto según la Secretaría de Planeación Municipal de Florida. Por su parte, la administración pública de Pradera estima que para abril de 2019 ha alcanzado un porcentaje de cumplimiento y ejecución del $75 \%$, dato suministrado por una evaluación interna hecha por la Secretaría de Planeación del municipio de Pradera.

No obstante, al entrevistar al grupo de funcionarios del municipio de Florida, se encuentra entre las observaciones a la ejecución del presupuesto la siguiente: 
Cada año se van reduciendo los recursos del presupuesto debido a contingencias ambientales como inundaciones y crecientes del río Cauca en Florida y Bolo en Pradera, eso obliga a las administraciones a mitigar el riesgo y reducir el daño. Bajo este panorama los recursos tienen una constante reasignación, y las dinámicas se entrecruzan y al mismo tiempo deben contar con la habilidad para responder de manera oportuna, coherente y canalizar las demandas sociales inmediatas producto de las crisis (funcionaria 4, grupo focal de abril de 2019).

Tanto en Pradera como Florida la constante demanda de derechos (individuales como sociales) por medios jurídicos a los cuales acuden las comunidades representa, para la visión de los funcionarios, un desgaste presupuestal y administrativo. "Hay una tensión entre administración pública y sociedad civil, pues se ha construido el imaginario en los cuadros de personal de la administración que la reclamación de los derechos ciudadanos y activar los mecanismos de participación dispuestos en la ley solo entorpece la función pública" (funcionaria 3, grupo focal de abril de 2019).

Las capacidades estatales externas no se centran en un relacionamiento fluido, sino conflictivo, a partir de las periódicas acciones jurídicas, como tutelas, derechos de petición y acciones populares. El interés sobre qué capacidades se tiene para hacer lo que se necesita y se proponga la administración pública y el gobierno local se centra en entender que estas son resultados logrados a través de la puesta en marcha de los objetivos que se traza la administración pública, es decir que se vinculan tanto las capacidades internas orientadas a la burocracia y las externas centradas en el relacionamiento con los actores del territorio.

Un hallazgo clave, relatado por el funcionario 1, en el grupo focal de abril de 2019, se remite a que los funcionarios no consideran que el problema sea de gestión para conseguir y ejecutar recursos, sino a los procesos y tiempos, ya que estos llegan a un cuello de botella que no permite realizar la gestión contractual de manera adecuada y oportuna. No hay una coordinación interadministrativa entre las diferentes dependencias que permita llevar a cabo la contratación de los profesionales por prestación de servicios, ni las obras civiles, ni los programas claves de la administración. 
La debilidad interinstitucional ocurre porque no hay una sincronización de los procesos y pasos que se necesitan para agilizarlos. Completa (2016) las denomina brechas de capacidad estatal, es justo cuando no hay un diálogo entre las diferentes áreas, departamentos o dependencias de una entidad y desemboca en que los procesos se vuelvan más lentos, es, por lo tanto, la separación entre la capacidad estatal y su desempeño.

Se concibe entonces una crisis de las capacidades estatales internas en las que Isuani (2012), refiriendo a Evans, plantea sobre el desempeño y la estructuración de la burocracia, y la complejidad para lograr una autonomía enraizada.

Dentro del conjunto de capacidades estatales internas surge, en el plano normativo y operativo, el debilitamiento de funciones misionales al servicio de la comunidad por parte de los directos responsables de la administración, al dedicar gran parte de su tiempo de gestión burocrática supervisando tareas de contratistas de prestación de servicios que vienen a suplir funciones propias de servidores públicos que debían estar nombrados en cargos de carrera administrativa. Esta debilidad institucional generalizada en todo el sector público de Colombia obedece a diversas razones, pero fundamentalmente se relaciona con el sistema clientelar arraigado en gran parte de los países de América latina.

En Florida como en Pradera hay una contratación por prestación de servicios por cada secretaría de entre 120 y 200 personas, según el funcionario 1, entrevistado en marzo de 2019. Esto obliga a los supervisores, que son los mismos secretarios del equipo del alcalde como directos responsables tanto en lo administrativo, disciplinario, como penalmente, a desentenderse de sus funciones misionales y de gestión para concentrarse en el control del cumplimiento de los objetivos de dichos contratos.

En el municipio de Florida hay 72 cargos de carrera administrativa, 26 vacantes y solo 5 han llegado por concurso de méritos en los últimos años, los demás trabajan en el municipio mediante contratos de prestación de servicios, creando así la llamada "nómina paralela". El problema radica en que no hay una conexión real entre todos los niveles del Estado, pues este viene diluyendo la responsabilidad de realizar los concursos de méritos para acceso a la carrera administrativa 
de una manera pertinente y oportuna que fortalezca la función de las administraciones locales (funcionaria 3, grupo focal de abril de 2019).

Las constantes reestructuraciones administrativas empezadas desde 2004 se han realizado "de manera amañada, favoreciendo a determinados individuos e intereses sin tener en cuenta las verdaderas necesidades de las comunidades, particularmente en un país diverso étnica y culturalmente", replica la funcionaria 4. Fernández y Vigil (2012) problematizan esta situación al plantear que los Estados latinoamericanos son débiles a pesar de ser grandes porque no son compactos en las formas de responder a las demandas sociales, e Isuani (2012), apoyándose en Weber, reseña la importancia del sistema de reclutamiento meritocrático que ha no ha sido eficaz ni eficiente en el caso del estudio abordado. Esta deficiencia de una burocracia consolidada y motivada se refleja en el interior de la institucionalidad en la ralentización de los procesos y el desorden interadministrativo; y en el exterior de la institucionalidad, se ve en la acepción conflictiva que existe entre la institucionalidad y una parte de los actores presentes en el territorio.

De acuerdo con los grupos focales realizados entre marzo y abril de 2019, en concreto se identifican cuatro problemáticas dentro de las dos administraciones de Florida y Pradera: 1) la gestión de contratación no es adecuada: los procesos se estancan de una dependencia a otra, los plazos que involucran la realización de los contratos por lo general no se cumplen; 2) no hay recurso humano tecnificado, calificado, preparado y motivado: los funcionarios públicos y contratistas en muchos casos carecen de formación técnica y profesional, así como de motivación para desempeñar funciones públicas; 3) los cambios de gobierno local afectan el funcionamiento de las administraciones, lo que implica la falta de continuidad en la implementación de políticas, planes y proyectos; y 4) los pocos funcionarios de carrera administrativa no se sienten motivados para mejorar la calidad de su gestión y no asumen la capacitación continua como una necesidad para su permanencia.

De otra parte, no hay una escisión clara entre las dinámicas electorales y las dinámicas de la administración pública, por lo tanto, la corriente política que gana las elecciones concentra el poder de contratación y gestión, invalidando otras posturas políticas y dado el caso que 
sea diferente a la del gobierno anterior se reversan muchos proyectos, planes, programas y estrategias, todo esto lo señala el funcionario 1. Se evidencian rupturas entre períodos de gobierno, lo que impide la construcción permanente de la memoria institucional y de las políticas a largo plazo. En respuesta y como mecanismo de resistencia, los funcionarios de carrera administrativa en muchas ocasiones ralentizan los procesos del alcalde que no sea a fin a su línea o partido político, es decir, se castiga desde el burócrata la victoria electoral del rival político, entorpeciendo la gestión de este y el cumplimiento de sus metas. Esta situación desemboca en una pérdida de eficacia y confianza, y es común en los dos municipios estudiados.

Conforme con lo anterior, las prácticas políticas habituales, como el clientelismo, afectan, por un lado, la efectividad y eficacia de la administración pública y los diferentes niveles de gestión; por el otro, es la forma de relacionarse que tienen el sector público con la comunidad, en la que se tranzan favores electorales por contratos o favores en la función pública. El factor político determina la pertinencia administrativa.

Según lo abordado, se han consolidado prácticas dentro de ambas administraciones en las que no hay un diálogo fluido entre dependencias y secretarías. Esto podría solventarse con robustecer una cultura organizacional de la calidad en la gestión del servicio público y en el fortalecimiento del talento humano en todos sus niveles, ya que redundaría en mayor penetración de la burocracia en las comunidades y territorios, y construiría a largo plazo las capacidades estatales tanto internas como externas de los municipios de Florida y Pradera. A este análisis fue el resultado al que los funcionarios presentes en el grupo focal de abril de 2019 llegaron luego de que se les indagó por el problema de la articulación dentro del sector público. Es decir, son conscientes de la falta de diálogo y engranaje del sector, sin embargo, las exigencias del día a día hacen que no se pueda abordar estructuralmente este problema. Ellos mismos lo relatan. "Como ejemplo, surge la construcción de un plan de ordenamiento territorial en el municipio de Florida en el año 2002, en el cual se dieron recomendaciones importantes sobre el territorio a cargo de expertos de la USAID. No obstante, se desechó ese documento en un período de gobierno porque el alcalde, sin estudios ni consideraciones técnicas, lo invalidó. Por lo tanto, el ejercicio quedó en anaqueles", menciona la funcionaria 3. 
Esta postura refleja la desidia que una parte del sector político tiene sobre los técnicos y expertos. "Invalidar ejercicios como la planificación, a mediano y largo plazo, y restarle importancia como herramienta gerencial es una práctica habitual en la cotidianidad administrativa de los municipios, orientados sobre todo a responder a las demandas y exigencias del día a día", continúa con el relato la funcionaria 3.

Sin embargo, la normatividad exige una planeación, básica y general, que es el documento del plan de desarrollo municipal; este se formula al inicio de cada período de gobierno, pero los funcionarios dicen que los territorios y las administraciones están en constante cambio, por lo que deberá centrarse en una planeación anual, según la percepción de ellos. Igualmente, hay intereses, exigencias y demandas de la comunidad, situaciones que las administraciones buscan solventar en el corto plazo. Pero la hipotética solución que plantean los funcionarios es la generación de un banco de proyectos territoriales donde cada año se priorice y se tomen decisiones para su ejecución, teniendo en cuenta su impacto, importancia y pertinencia.

En este panorama, las habilidades en cuanto a aspectos de tecnificación y desarrollo infraestructural son bajas y limitan sus respuestas frente a las demandas sociales. Es decir que las capacidades externas se ven limitadas por la maniobrabilidad de las capacidades internas.

La centralización también es un agravante que limita los márgenes de maniobra de las administraciones, pues, según los funcionarios en los grupos focales realizados en marzo y abril de 2019, cerca del $80 \%$ del presupuesto girado por el gobierno nacional debe observar la destinación específica que ordena el régimen legal. Y la poca planeación que se desarrolla está diseñada en el nivel central.

Igualmente, la evaluación con la que se examina el nivel de cumplimento de las metas y la eficacia en conseguirlas también es diseñada por el gobierno central, estandarizando todos los municipios independiente de la categoría a la cual pertenecen. Esta evaluación se centra en el producto cuantitativo, no se fija en indicadores de calidad, pertinencia, contextualización o cruce de diferentes datos y variables.

La diferencia entre la medición y la realidad es amplia, puesto que, al reducir todo a número y cantidad, y cuantificar los procesos, no se 
genera, según los funcionarios, un impacto real en las condiciones y calidad de vida de los habitantes de los territorios.

\section{Capacidades externas: en busca del vínculo con el territorio}

En procura de la construcción de capacidades estatales externas, los municipios, cuando están definiendo los distintos planes de desarrollo municipal, ${ }^{7}$ buscan mediante ejercicios participativos la voz de las comunidades, no obstante, en la enunciación final del plan no se alcanza a cubrir todas las necesidades sociales por limitaciones administrativas, temporales y económicas. "Ocurre entonces que se quedan por fuera demandas importantes y que las administraciones no alcanzan a cumplir", critica la funcionaria 6 en el grupo focal realizado en marzo de 2019.

Un escenario que vale la pena abordar es el concerniente a la multiculturalidad territorial y a la presencia de diversos actores sociales en los territorios. Hay una complejidad inherente a la relación entre actores sociales, sean indígenas, campesinos, afros, mujeres e, incluso, grupos armados ilegales.

En Florida existe una presencia de población indígena; en la zona montañosa, hay cinco comunidades indígenas reconocidas: dos cabildos indígenas: Cabildo Kwes Kiwe Yu y Cabildo San Juan Páez; y tres resguardos indígenas: Resguardo Indígena Nasa Kwes Kiwe, Resguardo Indígena Nasa Tha y Resguardo Indígena Triunfo Cristal Páez, lo que implica la búsqueda de consensos y diálogos de la administración pública frente a la autonomía de las comunidades indígenas.

“En este municipio, las estrategias de preservación de la identidad cultural que poseen las comunidades indígenas hacen que el acceso de la administración pública sea muy difícil, porque entran en tensión dos visiones de vida que al ejercer soberanía sobre el territorio yuxtaponen principios y valores, lo que desemboca en sospechas de ambas partes", menciona la funcionaria 6.

La implementación de planes y programas educativos y de salud "occidentales", por ejemplo, deben estar mediados por las autoridades indígenas, en el caso que se permitan hacer, ya que en la mayoría de

7 Cada que hay un nuevo gobierno municipal, es decir, cada cuatro años. 
las veces son bloqueados por la autoridad de la comunidad que es el gobernador indígena. Para los casos de educación sexual y reproductiva, planificación familiar y empoderamiento femenino, tanto el contenido como la intensidad deben estar reguladas, mediadas y coordinadas con las autoridades indígenas y deben comprender el visto bueno del gobernador, debido a que es parte de su autonomía. No obstante, no se generan diálogos fluidos ni negociaciones constantes para construir vínculos que permitan una armonización eficaz en la que la labor del Estado se realice y se preserve la cosmovisión de los pueblos indígenas (funcionaria 6, grupo focal de marzo de 2019).

Cualquier intervención del Estado y sus instituciones en estas zonas, y con principal énfasis en Florida, debe estar acompañada de solicitudes de permisos y autorizaciones dirigidas al gobernador y otorgadas por él. Las capacidades externas en este escenario se ponen a prueba, y es habitual que, dada la autonomía de las comunidades indígenas, los líderes de la comunidad establezcan relaciones directamente con entidades del gobierno central, deslegitimando al gobierno local al impedir o evitar el diálogo con este.

Las poblaciones de Florida como de Pradera han mostrado cambios incipientes y embrionarios de cultura democrática. No obstante, según la visión de los funcionarios públicos en el grupo focal de marzo de 2019, miembros de la comunidad aún siguen asistiendo a los talleres, actividades, ejercicios participativos y rendición de cuentas con la idea del refrigerio o por la comida, o porque van a reunirse con el alcalde, porque no hay nada más que hacer, porque le van a hacer oposición al gobierno local, y no por establecer acciones de empoderamiento ciudadano. Pero hay elementos que permiten divisar un pequeño cambio en estos comportamientos clientelistas y asistencialistas, un ejemplo es presenciar asistentes con habilidades técnicas y algún tipo de compromiso e interés en los asuntos públicos.

Los funcionarios, en el grupo focal de marzo de 2019, denotan la ausencia de procesos de participación ciudadana. Se concibe la participación como una estrategia planificada y metódica. Esta falencia se encuentra con la enunciada anteriormente relacionada con la planificación, dado que las estrategias de participación y los diálogos comunitarios se han realizado de manera posterior al diseño de la planeación. 
Al ser dinámicos los problemas sociales, estos se transforman, ajustan, amoldan y evolucionan de acuerdo con ciertas acciones e intereses, o incluso debido a la misma acción del Estado, que, al solucionar una problemática, deja entrever otras que han existido siempre pero no han sido tan visibles. Es por ello que hay un descrédito acerca de las administraciones municipales, se genera, por lo tanto, una división en la opinión pública que crea una suerte de polarización entre los que están a favor del alcalde y su gobierno y los que están en contra.

Para construir capacidades estatales tanto internas como externas, es preciso fortalecer la participación ciudadana, según reseñan los funcionarios de Florida y Pradera. La construcción de un trabajo colectivo en el que tanto los hechos reales y la realidad percibida vayan de la mano. Y ellos son conscientes de que el reto es potencializar la comunicación con la comunidad, es decir, establecer lo que Evans (1996) llama "burocracia incrustada". Desarrollar habilidades de penetración social por parte de una adecuada gestión y administración pública y de los recursos colectivos. Actividades institucionalizadas como la rendición de cuentas no son ejercicios efectivos en la construcción de ciudadanía y participación ciudadana, porque se precisa de un trabajo conjunto que asuma la suerte de estrategias de gobernanza para la consolidación de capacidades estatales tanto externas como internas.

De acuerdo con lo anterior, hay que resaltar el caso de Florida en el que el Consejo Comunitario de Cultura ha estado desarrollando un plan de fortalecimiento de manifestaciones artísticas y culturales que ha sido canalizado por la administración municipal. Todos los servicios de cultura del municipio están siendo impulsados por gestores culturales y por el Consejo Comunitario de Cultura, incluso la secretaria de esa cartera fue líder y gestora cultural.

Todos estos procesos están convirtiendo la oferta cultural de Florida en un referente tanto del municipio como de los demás poblados cercanos. En un ejercicio de participación y en el marco del fortalecimiento de capacidades estatales externas, se ha propuesto el Pacto de Cultura Ciudadana, en el cual los gestores culturales se reunieron para delimitar la base cultural; en el futuro tanto la administración como la comunidad piensan desarrollar el Plan Decenal de Cultura para 
fortalecer todas las ofertas artísticas y aportar a la construcción de paz (funcionaria 5, grupo focal de marzo de 2019).

Pradera cuenta desde 1992 con el Comité Comunitario de Desarrollo Local, donde confluyen líderes sociales, como los presidentes de corregimientos, diversas asociaciones de comunidades rurales, y en el mencionado comité se diseñan estrategias de integración. "También existe el Comité Técnico de Paz y Reconciliación creado en 2017, el cual se ha reunido tres veces, allí asisten grupos de mujeres de las FARC y movimientos como el LGTB", complementa el funcionario 2 a la intervención de la funcionaria 5 en el grupo focal de marzo de 2019.

Por otra parte, el Plan de Desarrollo 2016-2019 contiene un tema del posconflicto, el cual incluye un diagnóstico de la situación de las víctimas y un análisis a la situación rural del municipio, haciendo hincapié en el bajo desarrollo del campo en materia de infraestructura y productividad.

El municipio de Pradera no cuenta con una estrategia específica de construcción de confianza, y sus funcionarios consideran que existe un acercamiento y comunicación entre la administración pública y la burocracia con la comunidad, porque los miembros de la Secretaría de Gobierno y Agricultura hacen parte de las zonas rurales y de estos enclaves societales. También, el Plan de Desarrollo Municipal fue concertado con los diferentes actores sociales y legales que habitan el territorio, lo que constituye elemento generador de confianza a través de la participación comunitaria. Precisamente el eslogan de la actual administración es "Inclusión social".

Se plantean algunas acciones frente a las víctimas, como la reparación psicológica y el estudio de necesidades, acompañado de algunas ayudas económicas, trabajo que se realiza con la Unidad de Víctimas. En el marco de estas acciones, se cuenta con la realización de talleres de terapia colectiva para el posconflicto, disposición de transportes colectivos tradicionales como la "chiva", iniciativas empresariales y productivas para café y demás productos agrícolas relevantes, entre otras. Igualmente, la Unidad de Víctimas ha apoyado la feria de las truchas, con el objetivo de impulsar la silvicultura. Y un elemento por destacar es que en Pradera está la Oficina para la Víctimas, la cual es única y 
existe un asesor para el posconflicto de la administración (funcionario 2, en entrevista realizada en abril de 2019).

En el marco de las estrategias diseñadas para generar desarrollo en un municipio de vocación eminentemente rural como Pradera, uno de los proyectos principales para dar un nivel de integración urbanorural ha sido el fortalecimiento de la plaza de mercado, pues se piensa rediseñar para que la población campesina pueda traer sus alimentos al casco urbano, a fin de que no se siga trayendo de las centrales mayoritarias (Cavasa).

Ampliando la discusión sobre el relacionamiento multinivel dentro del Estado y su impacto concreto en los territorios, se ha indagado acerca de los planes y proyecto nacionales relacionados con la reforma rural integral, punto número uno del Acuerdo Final. Conforme a los funcionarios de la Alcaldía de Pradera, se reconoce que existen recursos de apalancamiento del orden nacional (de varias entidades) y también del departamento del Valle del Cauca y propios del municipio destinados a la reforma rural integral.

En este panorama, se ejemplifica la manera en que la Secretaría de Desarrollo Local coordina en materia de alimentación el Plan de Seguridad Alimentaria, que ha sido destacado por la Gobernación y tiene varios años en funcionamiento (quedó en los acuerdos del Concejo de 2015 y 2016). Opera intersectorialmente, incluida la Fundación Caicedo Rosales, la cual trabaja en gestión del conocimiento. Se hace, igualmente, mención al Pacto de Milán, donde Cali, Palmira, Pradera y Florida se unen (asociatividad) para comercializar productos agrícolas y que se queden en Cali.

Sin embargo, en el Plan Municipal de Desarrollo 2016-2019, además del diagnóstico, no se encuentra concretamente una verdadera estrategia encaminada a la implementación del punto uno del Acuerdo Final. No obstante, hay intenciones de fortalecer el sector agrícola como vehículo de sostenimiento de la población rural. En especial sobre los jóvenes, pues estos están migrando hacia las grandes ciudades, relata con preocupación el funcionario 2 en la entrevista efectuada en marzo de 2019.

En cuanto al tema concerniente a la implementación de los Acuerdos de Paz, la estrategia usada por los programas de desarrollo con 
enfoque territorial ha generado muchas expectativas en las comunidades indígenas, campesinas y afrodescendientes, porque han propuesto alrededor de 207 iniciativas y proyectos; sin embargo, de esos, para la región del "Norte del Cauca-Alto Patía", por parte de Florida y Pradera quedaron solo 5..$^{8}$ Las estrategias usadas por la Agencia de Renovación del Territorio orientaron a que las comunidades demandaran acciones de acuerdo con sus necesidades, generando una amplia esperanza en todas las comunidades, mencionan unánimemente todos los funcionarios participantes en los grupos focales de marzo y abril de 2019.

Así mismo, el Consejo de Paz del municipio de Florida está capacitado e interesado en la construcción de los proyectos y en aplicar a convocatorias de cooperación internacional, que son complejas y complicadas de cumplir.

En Florida se han generado nuevas dinámicas de disputa entre actores ilegales. Han incursionado nuevos grupos armados que antes no habían hecho presencia, han surgido nuevos peligros en los territorios por parte de las disidencias de las FARC, y los indígenas, al rechazar la presencia de cualquier actor armado en sus territorios, rechazan sobre todo a las Fuerzas Militares, mientras quedan a merced de las fuerzas ilegales, lo que produce que se hayan consolidado acuerdos de control territorial entre los grupos armados y las comunidades campesinas. Los cultivos de uso ilícito se han potencializado y fortalecido por la no presencia del Ejército. Además, las amenazas, amedrentamientos e, incluso, asesinatos de líderes sociales se han vuelto a activar (funcionaria 7 , grupo focal de marzo de 2019).

En contraste, Pradera responde a otra dinámica diferente, pues el control territorial en la zona rural es mayor por parte de las fuerzas del Estado, las comunidades indígenas han respondido diferente en comparación con las de Florida y han consentido el acceso tanto del Ejército como de las demás entidades del Estado, permitiendo el acercamiento de la institucionalidad, compara la funcionaria 5.

Pradera cuenta con el reconocimiento del Resguardo Indígena Kwet Wala en la parte alta. La presencia del único resguardo indígena en Pradera centraliza la negociación que hace la administración pública con la

8 Dato otorgado por los funcionarios de la Alcaldía de Florida. 
comunidad indígena en general; igualmente, la presencia de habitantes es menor, o sea, hay menos población de este pueblo que en Florida. Ese aspecto diferenciador hace que las lógicas de penetración de la administración pública en el territorio sean más eficaces, y problemáticas como la presencia de actores armados ilegales y cultivos de uso ilícito sean más rápidamente contenidas por las fuerzas del Estado.

$Y$, en esta línea, los funcionarios consultados tanto en las entrevistas como en los grupos focales realizados en marzo y abril de 2019 enuncian que la administración pública apoya iniciativas de la comunidad indígena presente en el municipio, ya que existen proyectos priorizados para ellos. En un ejercicio democrático, el Resguardo Indígena Kwet Wala tiene su plan de vida y se ha articulado al Plan de Desarrollo $\mathrm{Mu}^{-}$ nicipal 2016-2019. Para el caso de la población afro que está ubicada en el casco urbano, se les apoya en asuntos culturales a través de tema de víctimas, pues son migrantes de otras regiones que han padecido el conflicto. En ese conjunto de acciones y estrategias para vincular a las diferentes comunidades, se celebra el Día Agro, Día del Campesino. Eventos en los que hay presencia tanto de afros como de indígenas y población rural. En síntesis, los funcionarios de Pradera plantean que sí existe reconocimiento de los diferentes grupos étnicos.

No obstante, es de reseñar que los funcionarios consultados no dan respuestas concretas ni puntuales acerca de la reforma rural integral, las zonas de reserva campesinas, fondo y acceso y garantía de seguridad a la tierra, Sistema General de Información del Catastro Multipropósito, como estrategias nacionales para la implementación de reformas orientadas a la construcción de paz, en particular, y de Estado, en general.

\section{Conclusiones}

Conforme con el propósito general de este trabajo, que es analizar la capacidad estatal de los municipios de Pradera y Florida para una agenda de desarrollo local resultado del Acuerdo Final para la paz entre el gobierno colombiano y la guerrilla de las FARC-EP, se concibe que las capacidades estatales, como referente conceptual, están enmarcadas en una visión relacional. 
Por lo tanto, las administraciones municipales de Florida y Pradera, como el nivel más inmediato del Estado en el territorio, y con el fin de llevar a cabo una agenda de desarrollo local, están orientadas a generar un fortalecimiento de sus relaciones tanto entre ellas y con demás entidades del Estado, es decir, a nivel burocrático -capacidades internas-, como hacia las comunidades y territorios en los que pretenden penetrar e influir -capacidades externas-.

Las capacidades estatales que se precisan para la construcción de paz van más allá del acuerdo logrado entre el gobierno nacional y las FARC en 2016, se centran en niveles más locales y en habilidades más cotidianas: el mérito de los funcionarios públicos, mejorar los incentivos de quienes están en la administración pública, la consolidación de cooperación interinstitucional entre las diferentes áreas del gobierno local y en los distintos niveles del Estado, la preservación de la memoria institucional, la continuación de estrategias exitosas a través del tiempo. El 'tipo ideal' de la burocracia weberiana y del servicio público no se refleja en la realidad estudiada en Florida y Pradera (Valle), porque la burocracia no crea incentivos en el personal de carrera administrativa; los gobiernos locales usan la contratación particular y limitada para intentar implementar sus metas; y el nivel de inserción de la institucionalidad en el territorio es bajo.

La cimentación de una relación sólida entre el gobierno local y la comunidad es primordial para llevar a cabo las capacidades estatales externas. Se muestra que en Florida las condiciones de este relacionamiento son conflictivas y tensas con los indígenas, pero en el sector cultural se han realizado ejercicios interesantes en los que se teje capital social y se logra una articulación entre la institucionalidad y la comunidad.

En Pradera, las relaciones entre la administración municipal y las comunidades indígenas son más fluidas, y la presencia del Estado es diferenciada con respecto a Florida. Esta vinculación con las poblaciones hace que haya una manifestación, todavía muy incipiente, de la burocracia incrustada que permite referenciar al gobierno local como un actor clave que puede responder positivamente a las demandas sociales. Sin embargo, la capacidad de penetración y la fortaleza institucional deben consolidarse más. 
Autores como Completa (2016), Bertranou (2013), Rodríguez (2004) y Mann (1984) conciben las dos dimensiones de las capacidades estatales, siendo estás internas y externas. Las que se han mencionado en los párrafos anteriores son las que se podrían entender como internas, puesto que procuran que la burocracia sea eficiente. Para Aguilar (2010) y Evans (1996), la administración solo es valorada en virtud de sus resultados y la burocracia debe estar profesionalizada para incrustarse y enraizarse con sus círculos sociales de influencia. Igualmente, se da cuenta de las condiciones de relacionamiento que la institucionalidad ha construido con sus territorios y comunidades más cercanas, como lo son los indígenas y los habitantes sin ninguna inscripción étnica particular pero preocupados por sus territorios y por los asuntos que allí conciernen.

\section{Referencias}

Aguilar, L. (2010). Gobernanza, el nuevo proceso de gobernar. México: Ed. Fundación para la Libertad.

Alonso, G. (Ed.). (2007). Elementos para el análisis de capacidades estatales. En Capacidades estatales, instituciones y política social (pp. 17-40). Editorial Prometeo.

Bertranou, J. (septiembre, 2013). Capacidad estatal: aportes al debate conceptual. Ponencia presentada en el Séptimo Congreso Argentino de Administración Pública, Mendoza.

Blanco, A., \& Arrieta, J. (2019). Presencia diferenciada del Estado: el necropoder y las FARC en Colombia. Justicia, 24(36), 1-13.

Colombia, Presidencia de la República. (2016). Acuerdo final para la terminación del conflicto y la construcción de una paz estable y duradera.

Colombia, Presidencia de la República. (2017). Programa de Desarrollo con Enfoque Territorial (PDET).

Completa, E. (2016). Capacidad estatal, brechas de capacidad y fortalecimiento institucional. Análisis Político, (87), 60-76.

Completa, E. (2017). Capacidad estatal: ¿qué tipo de capacidades y para qué tipo de Estado? Revista POSTData: Revista de Reflexión y Análisis Político, 22(1), 111-140.

Evans, P. (1996). El Estado como problema y como solución. Desarrollo Económico, 35(140), 529-562. 
Fernández, V., \& Vigil, J. (2012). Capacidades estatales regionales: consideraciones teóricas y metodológicas para su análisis en América Latina. Revista de Políticas Públicas, 16(1), 51-65.

Garay, L., Salcedo, E., Isaac de León, A., \& Guerrero, B. (2008). La captura y reconfiguración cooptada del Estado en Colombia. Bogotá: Ed. Método.

García Villegas, M., Torres Echeverry, N., Revelo Rebolledo, J., Espinosa Restrepo, J., \& Duarte Mayorga, N. (2016). Los territorios de la paz. La construcción del Estado local en Colombia. Dejusticia.

Gilio, A. (2016). Desarrollo de capacidades estatales para gobiernos locales: dimensiones e indicadores para su diagnóstico. Revista del CLAD Reforma y Democracia, (66), 228-258.

Hilderbrand, M., \& Grindle, M. (1997). Building sustainable capacity in the public sector. What can be done? Boston: Harvard University Press.

Isuani, F. (2012). Instrumentos de políticas públicas. Factores claves de las capacidades estatales. DAAPGE, (19), 51-74.

López, C. (2010). Y refundaron la patria... de cómo mafiosos y políticos reconfiguraron el Estado colombiano. Bogotá: Penguin Random House.

Mann, M. (1984). The autonomous power of the State. Archives Européennes de Sociologie, 25, 185-213.

Ospina, S. (2002). Construyendo capacidad institucional en América Latina: el papel de la evaluación como herramienta modernizadora. Ponencia presentada en el VII Congreso Internacional del CLAD sobre la Reforma del Estado y de la Administración Pública, Lisboa.

Oszlak, O., \& Orellana, E. (1991). El análisis de la capacidad institucional: aplicación de la metodología SADCI.

Restrepo, D. (2018). Dilemas y retos del enfoque territorial en la implementación de los acuerdos de paz. Función Pública.

Rey, M. (2014). Capacidad estatal y poder del Estado en la Latinoamérica del siglo XXI: una perspectiva política para el análisis de las políticas públicas y la estatalidad. Revista Estado y Políticas Públicas, (2), 115-139.

Rodríguez-Gustá, A. (2004). Capacidades estatales: reflexiones en torno a un programa de investigación. Ponencia presentada en VI Jornadas de Sociología, Facultad de Ciencias Sociales, Universidad de Buenos Aires.

Sarria, C. (2015). ¿Las agencias son unidades administrativas especiales? Revista Digital de Derecho Administrativo, (13), 237-252.

Uribe López, M. (2018). Infraestructuras de paz y estatalidad en Colombia. Revista Perfiles Latinoamericanos, 26, 167-189. 